\title{
Cotorsion radicals and projective modules
}

\author{
John A. Beachy
}

\begin{abstract}
We study the notion (for categories of modules) dual to that of torsion radical and its connections with projective modules.
\end{abstract}

Torsion radicals in categories of modules have been studied extensively in connection with quotient categories and rings of quotients. (See [8], [12] and [13].) In this paper we consider the dual notion, which we have called a cotorsion radical. We show that the cotorsion radicals of the category $R^{M}$ correspond to the idempotent ideals of $R$. Thus they also correspond to TTF classes in the sense of Jans [9].

It is well-known that the trace ideal of a projective module is idempotent. We show that this is in fact a consequence of the natural way in which every projective module determines a cotorsion radical. As an application of these techniques we study a question raised by Endo [7], to characterize rings with the property that every finitely generated, projective and faithful left module is completely faithful. We prove that for a left perfect ring this is equivalent to being an S-ring in the sense of Kasch. This extends the similar result of Morita [15] for artinian rings.

\section{Cotorsion radicals}

Throughout this paper $R$ will denote an associative ring with identity, and $R^{M}$ and $M_{R}$ will denote the categories of unital left and right $R$-modules, respectively. Homomorphisms will be written on the right. The reader is referred to Lambek [11] for basic definitions. 
Following the terminology of Maranda [13], a subfunctor $\rho$ of the identity on an abelian category $A$ will be called a preradical of $A$. $A$ preradical $\rho$ is said to be proper if $\rho(A) \neq A$ for some $A \in A$ and $\rho\left(A^{\prime}\right) \neq 0$ for some $A^{\prime} \in A$. A preradical $\rho$ of $R^{M}$ is proper if and only if $\rho(R) \neq R$ and $\rho(M) \neq 0$ for some $M \in R^{M}$. A preradical $\rho$ is called idempotent if $\rho^{2}=\rho$; it is called a torsion preradical if $\rho$ is a left exact functor; it is called a radical if $\rho(A / \rho(A))=0$ for all $A \in A$. An object $A \in A$ is $\rho$-torsion if $\rho(A)=A$ and $\rho$-torsionfree if $\rho(A)=0$.

In order to establish notions formally dual to these, we note that a preradical $\rho$ of $R^{M}$ associates with each module $R^{M}$ a short exact sequence

$$
0 \rightarrow \rho(M) \rightarrow M \rightarrow M / \rho(M) \rightarrow 0
$$

and with each $R$-homomorphism $f: R^{M} \rightarrow{ }_{R}^{N}$ a morphism of short exact sequences

$$
\begin{aligned}
& 0 \rightarrow \rho(M) \rightarrow M \rightarrow M / \rho(M) \rightarrow 0 \\
& 0 \rightarrow \rho(N) \rightarrow N \rightarrow N / \rho(N) \rightarrow 0
\end{aligned}
$$

where the endomorphisms are those induced by $f$. (Since $\rho$ is a preradical we must have $(\rho(M)) f \subseteq \rho(N)$.$) As in [4], we denote by 1 / \rho$ the functor which assigns to each module $R^{M}$ the module $M / \rho(M)$ and to each $R$-homomorphism $f$ the homomorphism induced by $f$ in the diagram above.

If we use * to denote the dual of $1 / \rho$ in the dual category $R^{M *}$ of $R^{M}$, then $(1 / \rho)^{*}$ is a preradical, and since $\rho$ is a radical if and only if $(1 / \rho)^{2}=1 / \rho$, it follows that $\rho$ is a radical if and only if $(1 / \rho)^{*}$ is an idempotent preradical. Furthermore, $\rho$ is an idempotent preradical if and only if $(1 / \rho)^{*}$ is a radical, so these two notions are dual.

PROPOSITION 1.1. Let $\rho$ be a preradical of $R^{M}$. The following conditions are equivalent: 
(i) $(1 / \rho)^{*}$ is a torsion radical of $k^{M *}$;

(ii) $\rho$ is idempotent and $1 / \rho$ is right exact;

(iii) $\rho$ is idempotent and every epimorphism $\pi:{ }_{R}{ }^{M} \rightarrow{ }_{R}^{N}$ induces an epimorphism $\rho(\pi): \rho(M) \rightarrow \rho(N)$.

Proof. Conditions ( $i$ ) and (ii) are easily seen to be equivalent using the definitions and remarks preceding the proposition. To show the equivalence of ( $i$ i) and ( $i i i)$, given an exact sequence $0 \rightarrow M^{\prime} \rightarrow M \rightarrow M^{\prime \prime} \rightarrow 0$ in $R^{M}$, consider the induced diagram:

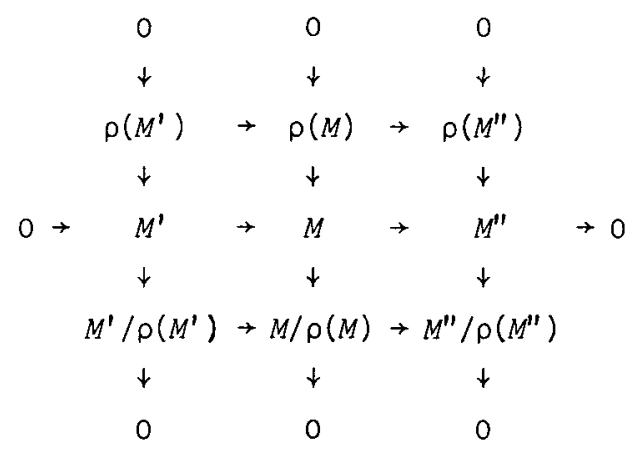

In the diagram the columns are exact and $M / \rho(M) \rightarrow M^{\prime \prime} / \rho\left(M^{\prime \prime}\right)$ is an epimorphism. A standard diagram chasing argument shows that

$$
M^{\prime} / \rho\left(M^{\prime}\right) \rightarrow M / \rho(M) \rightarrow M^{\prime \prime} / \rho\left(M^{\prime \prime}\right)
$$

is exact at $M / \rho(M)$ if and only if $\rho(M) \rightarrow \rho\left(M^{\prime \prime}\right)$ is an epimorphism. This completes the proof of the proposition. //

It is clear that a preradical which satisfies condition (iii) of the above proposition must be a radical, and so we make the following definition.

DEFINITION 1.2. A preradical $\rho$ in $R^{M}$ will be called a cotorsion radical if $\rho$ is idempotent and every epimorphism $\pi:{ }_{R} M \rightarrow{ }_{R} N$ induces an epimorphism $\rho(\pi): \rho(M) \rightarrow \rho(N)$.

PROPOSITION 1.3. Let $\rho$ be a preradical of $k^{M}$. The following conditions are equivalent:

(i) every epimorphism $\pi:{ }_{R} M \rightarrow{ }_{R} N$ induces on epimorphism 


$$
\begin{aligned}
\rho(\pi) & : \rho(M) \rightarrow \rho(N) ; \\
\text { (ii) } \rho(M) & =\rho(R) \cdot M \text { for alz } M \in R^{M} ;
\end{aligned}
$$

(iii) $\rho$ is a radical and every factor module of a $\rho$-torsionfree module is $\rho$-torsionfree.

Proof. (i) $\Rightarrow$ (ii). Let $M \in R^{M}$ and let $R^{I}$ be the direct sum of $I$ copies of $R$, where the index set $I$ is just $M$ itself. Then the homomorphisms $f_{m}: R \rightarrow M$ defined for all $m \in M$ by $(r) f_{m}=r m$ together define an epimorphism $f: R^{I} \rightarrow M$. Since $\rho\left(R^{I}\right)$ consists precisely of those elements of $R^{I}$ for which each component belongs to the ideal $\rho(R)$, it follows on assuming condition (i) that

$$
\rho(M)=\left(\rho\left(R^{I}\right)\right) f=\rho(R) \cdot M \text {. }
$$

(ii) $=$ (iii). For all $M \in{ }_{R^{M}}, \rho(R) \cdot(M / \rho(R) \cdot M)=0$, and if $\rho(R) \cdot M=0$, then $\rho(R) \cdot M^{\prime \prime}=0$ for every factor module $M^{\prime \prime}$ of $M$.

(iii) $\Rightarrow(i)$. Let $\pi: R^{M} \rightarrow{ }_{R}^{N}$ be an epimorphism. Then $\pi$ induces an epimorphism $M / \rho(M) \rightarrow N /(\rho(M)) \pi$, and so if condition (iii) is satisfied, $M / \rho(M)$ is $\rho$-torsionfree and $N /(\rho(M)) \pi$ must be $\rho$-torsionfree as well. It follows that we must have $\rho(N) \subseteq(\rho(M)) \pi$, and therefore $\rho(N)=(\rho(M)) \pi$. $\quad / /$

The above proposition generalizes a result of Kurata [10, Lemma 2.1]. Moreover, if $A$ is any ideal of $R$, the proof shows that setting $\rho(M)=A \cdot M$ for all $M \in R^{M}$ defines a preradical of $k^{M}$ which satisfies the conditions of Proposition 1.3. It follows immediately that there is a one-to-one correspondence between ideals of $R$ and radicals of $R^{M}$ satisfying the conditions of Proposition 1.3. (Note that for such a radical $\rho$ the $\rho$-torsionfree $R$-modules are nothing more than the left $R / \rho(R)$-modules.) In this situation the radical $\rho$ is idempotent if and only if $\rho(R)$ is an idempotent ideal, so we have proved the following theorem.

THEOREM 1.4. There is a one-to-one correspondence between cotorsion 
radicals of $R^{M}$ and idempotent ideals of $R$.

A class $T$ of modules is called by Jans [9] a TTF class if it is closed under taking submodules, factor modules, direct products, and if in any exact sequence $0 \rightarrow M^{\prime} \rightarrow M \rightarrow M^{\prime \prime} \rightarrow 0, M^{\prime}, M^{\prime \prime} \in T$ implies $M \in T$. A TTF class is the torsionfree class of a cotorsion radical, and conversely the torsionfree class of a cotorsion radical is a TTF class, so Theorem 1.4 gives another proof of Corollary 2.2 of Jans [9], that TTF classes correspond to idempotent ideals.

If $M, N \in R^{M}$, we denote by $\operatorname{tr}_{N}(M)$ the sum in $N$ of all R-homomorphic images of $M$. The functor $\operatorname{rad}^{M}$ defined by $\operatorname{rad}^{M}(N)=\operatorname{tr}_{N}(M)$, for all $N \in R^{M}$, is an idempotent preradical. The following proposition gives a new way of viewing some known results concerning the trace ideal of a projective module. (See the appendix in the paper by Auslander and Goldman [1].)

PROPOSITION 1.5. If ${ }_{R}^{P}$ is projective, then rad ${ }^{P}$ is a cotorsion radical.

Proof. For $N \in R^{M}, \operatorname{rad}^{P}(N)=0 \Leftrightarrow \operatorname{hom}_{R}(P, N)=0$. If $P$ is projective, then it follows that $\operatorname{hom}_{R}\left(P, N / \operatorname{rad}^{P}(N)\right)=0$ for all $N \in R^{M}$, since any non-zero homomorphism $f: P \rightarrow N / \mathrm{rad}^{P}(N)$ lifts to a homomorphism $g: P \rightarrow N$ with $(P) g \notin \operatorname{rad}^{P}(N)$, a contradiction. Furthermore, if $\operatorname{hom}_{R}(P, N)=0$, then $\operatorname{hom}_{R}\left(P, N^{\prime \prime}\right)=0$ for every factor module $N^{\prime \prime}$ of $N$. We may conclude from Proposition 1.3 ( $i$ ii) that $\rho$ is a cotorsion radical. $/ /$

A partial converse to Proposition 1.5 can be obtained. A module $R^{M}$ is called torsionless if it $c$ an be embedded in a direct product of copies of $R^{R}$. Any projective module is torsionless, and any submodule of a torsionless module is torsionless.

PROPOSITION 1.6. If $\rho$ is a cotorsion radical of $k^{M}$, then 
$\rho=\operatorname{rad}^{M}$ for a torsionless module ${ }_{R}^{M}$.

Proof. If $\rho$ is a cotorsion radical of $R^{M}$, let $R^{M}=\rho(R)$. As a left ideal, $\rho(R)$ is torsionless, and since $\rho$ is idempotent, it is $\rho$-torsion. Therefore we must have $(M) f \subseteq \rho(N)$, for all $N \in R^{M}$ and $f \in \operatorname{hom}_{R}(M, N)$. Thus $\operatorname{rad}^{M}(N) \subseteq \rho(N)$. On the other hand, $\rho(N)=\rho(R) \cdot N \subseteq \operatorname{tr}_{N}(\rho(R))=\operatorname{rad}^{M}(N)$, for all $N \in{ }_{R}^{M}$. //

If $\pi:{ }_{R}^{P} \rightarrow{ }_{R}^{M}$ is an epimorphism, with $P$ projective, then $P$ is called a projective cover of $M$ if $\operatorname{ker}(\pi)$ is small in $P$, that is, if for all submodules $P^{\prime}$ of $P, P=P^{\prime}+\operatorname{ker}(\pi)$ implies $P^{\prime}=P$. The notion of projective cover was defined and studied by Bass [3]. He called a ring $R$ left perfect if every left $R$-module has a projective cover.

LEMMA 1.7. Let $R^{M}$ be a module with a projective cover $\pi:{ }_{R}^{P} \rightarrow{ }_{R}^{M}$. If $\rho$ is a cotorsion radical of $R^{M}$ and $M$ is $p$-torsion, then $P$ is $\rho$-torsion.

Proof. The proof depends only on the fact that $k e r(\pi)$ is small. If $M$ is $p$-torsion, then $\rho(M)=M$, and since $\rho$ is a cotorsion radical, we must have $(\rho(P)) \pi=\rho(M)=M$. Thus $\rho(P)+\operatorname{ker}(\pi)=P$, and since $\operatorname{ker}(\pi)$ is small, this implies that $\rho(P)=P$. $/ /$

THEOREM 1.8. If every idempotent ideal of $R$ has a projective cover as a left R-module, then every cotorsion radical of $R^{M}$ is of the form $\operatorname{rad}^{P}$ for a projective module ${ }_{R}^{P}$.

Proof. Assume the given condition and let $\rho$ be a cotorsion radical, $M=\rho(R)$, and $P \rightarrow M$ be a projective cover of $M$. By Proposition 1.6, $\rho=\operatorname{rad}^{M}$, and since $M$ is a homomorphic image of $P$ it follows that $\rho(N)=\operatorname{rad}^{M}(N) \subseteq \operatorname{rad}^{P}(N)$, for all $N \in R^{M}$. By Lemma 1.7, $P$ is $\rho$-torsion, and therefore $\operatorname{rad}^{P}(N) \subseteq \rho(N)$, for all $N \in R_{R}^{N}$. //

We note that the condition of Theorem 1.8 is satisfied in any of the following cases: 
(i) $R$ is left perfect;

(ii) $R$ is left semi-perfect and left noetherian;

(iii) $R$ is left hereditary.

(Recall that a ring $R$ is left semi-perfect if every finitely generated left $R$-module has a projective cover, and left hereditary if every left ideal is projective.)

\section{Faithful preradicals}

DEFINITION 2.1. A preradical $\rho$ of $R^{M}$ will be called faithful if $\rho(M)$ is faithful for every faithful module $R^{M}$.

Recall that $R^{M}$ is faithful if the annihilator ideal $\operatorname{ann}(M)=\{r \in R \mid r M=0\}$ is the zero ideal. For a left ideal $A$ of $R$ we write $Z(A)$ rather than $\operatorname{ann}(A)$.

PROPOSITION 2.2. Let $\rho$ be a preradical of $R^{M}$. Then $\rho$ is faithful if and only if $Z(\rho(R))=0$.

Proof. ONLY IF: This is obvious from the definition.

IF: If $Z(\rho(R))=0$ and $R^{M}$ is faithful, then $\rho(R) \cdot M$ is faithful. Since $\rho(M) \supseteq \rho(R) \cdot M$ it is clear that $\rho(M)$ is faithful. //

The ring $R$ is prime if for any ideals $A, B$ of $R, A \cdot B=0$ implies $A=0$ or $B=0$. It follows from Proposition 2.2 that if $R$ is prime then a preradical $\rho$ is faithful if and only if $\rho(R) \neq 0$. In fact, this property characterizes prime rings.

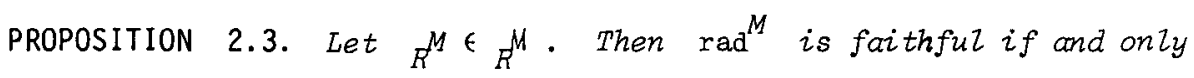
if $M$ has a faithful, torsionless factor module.

Proof. ONLY IF: If $\operatorname{rad}^{M}$ is faithful, let $M^{\prime}$ be the intersection of all kernels of homomorphisms from $R^{M}$ to $R^{R}$, and $M^{\prime \prime}=M / M^{\prime}$. Then $M^{\prime \prime}$ is torsionless and $\operatorname{tr}_{R}(M)=\operatorname{tr}_{R}\left(M^{\prime \prime}\right)$. Since $\operatorname{rad}^{M}$ is faithful we have $Z\left(\operatorname{tr}_{R}\left(M^{\prime \prime}\right)\right)=0$, and a short argurnent can be given to show that $M^{\prime \prime}$ must therefore be faithful. 
IF: Suppose that $M$ has a faithful, torsionless factor module $M^{\prime \prime}$. Then $\operatorname{tr}_{R}\left(M^{\prime \prime}\right) \subseteq \operatorname{tr}_{R}(M)$, so it suffices to show that $l\left(\operatorname{tr}_{R}\left(M^{\prime \prime}\right)\right)=0$. Since $M^{\prime \prime}$ is faithful, given $0 \neq r \in R$ there exists $m \in M^{\prime \prime}$ with $r m \neq 0$, and then since $M^{\prime \prime}$ is torsionless there exists $f \in \operatorname{hom}_{R}\left(M^{\prime \prime}, R\right)$ such that $(m) f \neq 0$. But then $r((m) f) \neq 0$, and $(m) f \in \operatorname{tr}_{R}\left(M^{\prime \prime}\right)$. //

Azumaya [2] calls a module $R^{M}$ completely faithful if $\operatorname{tr}_{R}(M)=R$. The next proposition is related to $\$ 3$ on faithful projective modules, but it is stated here in terms of cotorsion radicals. It follows from the correspondence between cotorsion radicals and idempotent ideals that $R^{M}$ has no proper faithful cotorsion radicals if and only if $Z(A) \neq 0$ for every proper idempotent ideal $A$ of $R$. If, moreover, every cotorsion radical is of the form $\operatorname{rad}^{P}$ for a projective module ${ }_{R}^{P}$, then by Proposition 2.2 and Proposition 2.3 this condition is equivalent to the condition that every faithful, projective left $R$-module is completely faithful. A ring $R$ is called a left $S$-ring if $Z(A) \neq 0$ for every proper right ideal $A$ of $R$.

PROPOSITION 2.4. Let $R$ be a left perfect ring. Then $R^{M}$ has no proper faithful cotorsion radicals if and only if $R$ is a left $S$-ring.

Proof. IF: If $R$ is a left S-ring, then certainly $Z(A) \neq 0$ for every proper idempotent ideal $A$, and the result follows from Theorem 1.4 .

ONLY IF: Lambek [11, Chapter 4] calls a right ideal $D$ of $R$ dense if for all $f \in \operatorname{hom}_{R}\left(R, E\left(R_{R}\right)\right), f=0$ if $(D) f=0$, where $E\left(R_{R}\right)$ is the injective envelope of the right $R$-module $R_{R}$. Equivalently, $D$ is dense if and only if $\operatorname{hom}_{R}\left(R / D, E\left(R_{R}\right)\right)=0$.

Jans, in Theorems 2.1 and 3.1 of [9], has shown that if $R$ is left perfect, then the intersection $D_{0}$ of all dense right ideals is a dense, idempotent ideal. Lambek has shown [11, Corollary, p. 96] that an ideal $D$ is dense as a right ideal if and only if $Z(D)=0$. Thus we must have $Z\left(D_{0}\right)=0$. Then from our assumption it follows that $D_{0}=R$, and by Theorem 3.2 of Jans [9], $R$ is a left S-ring. // 
Our proof depended only on the fact that the intersection of dense right ideals is again dense, which, as shown by Jans, holds for a larger class of rings than just the class of left perfect rings. Alin and Armendariz [O] and $\mathrm{Dlab}$ [5] have given proofs that in a left perfect ring the intersection of right ideals dense with respect to any torsion radical of $M_{R}$ is again dense. (A right ideal $D$ is dense with respect to a torsion radical $\rho$ of $M_{R}$ if $\rho(R / D)=R / D$.)

\section{Faithful projective modules}

Azumaya in [2] characterized rings for which every faithful left $R$-module is completely faithful. Endo [7, 56] investigated conditions under which every finitely generated, projective and faithful left $R$-module is completely faithful. We show in this section that if $R$ is left perfect then this condition is satisfied if and only if $R$ is a left $S$-ring. This generalizes a result of Morita [15, Theorem 1]. We first give some general results.

If $\frac{p}{R}$ is finitely generated and projective, then for some positive integer $n$ there exists an epimorphism $\pi: R^{n} \rightarrow P$, and this splits by a monomorphism $\theta: P \rightarrow R^{n}$. Since $\pi \theta$ is an endomorphism of a free module, it can be described by an $n \times n$ matrix $X$ with entries in $R$, and $X$ is idempotent. Let $\langle X\rangle$ denote the (two-sided) ideal generated by the entries of the matrix $X$. Although the matrix $X$ is not uniquely determined by $P$, the next lemma shows that the ideal $(X)$ is uniquely determined.

LEMMA 3.1. Let ${ }_{R}^{P}$ be finitely generated and projective, and $X$ an associated idempotent matrix. Then $\langle X\rangle=\operatorname{tr}_{R}(P)$.

Proof. We first show that $\langle X\rangle \subseteq \operatorname{tr}(P)$. Since $\operatorname{tr}_{R}(P)$ is an ideal, we only need to show that the generators of $\langle X\rangle$ are contained in $\operatorname{tr}_{R}(P)$. This follows immediately upon considering the projections $\left(R^{n}\right) X \rightarrow R^{n} \rightarrow R$, where $X: R^{n} \rightarrow P \rightarrow R^{n}$.

To show that $\operatorname{tr}_{R}(P) \subseteq\langle X\rangle$, it suffices to show that $(P) f \subseteq\langle X\rangle$ for 
all $f \in \operatorname{hom}_{R}(P, R)$. Any such $R$-homomorphism $f$ can be extended to $g: R^{n} \rightarrow R$, and then the components of this extension are determined by multiplication on the right by elements of $R$. Thus $(P) f=\left(R^{n}\right) X g$, and so $(P) f \subseteq\langle X\rangle$. //

The above lemma gives one method of characterizing trace ideals of finitely generated, projective modules. It can be extended to a characterization of trace ideals of arbitrary projective modules by using row-finite matrices. Since a projective module $R^{P}$ is faithful if and only if $l\left(\operatorname{tr}_{R}(P)\right)=0$ (Propositions 2.2 and 2.3 ), we have the following proposition.

PROPOSITION 3.2. Every finitely generated, projective and faithful left $R$-module is completely faithful if and only if $Z(A) \neq 0$ for every proper idempotent ideal $A$ of $R$ which is generated as a two-sided ideal by the entries of a finite idempotent matrix over $R$.

Although this characterization is somewhat unwieldy, it can be used to give a short proof of the well-known fact that if $R$ is a commutative ring, then every finitely generated, projective and faithful $R$-module is completely faithful. To show this, suppose that $X$ is a finite idempotent matrix with entries in a commutative ring $R$. Let $E$ be the identity matrix and let $E-X$ be the adjoint matrix of $E-X$. Since $X$ is idempotent, $(E-X)(X)=(0)$, and therefore $(\operatorname{det}(E-X))(X)=\widehat{(E-X)}(E-X)(X)=(0)$. If $z(\langle X))=0$, then this implies that $\operatorname{det}(E-X)=0$, which shows that $I \in\langle X\rangle$ and $\langle X\rangle=R$. Thus, in a commutative ring, any proper ideal generated by a finite idempotent matrix must have non-zero annihilator.

The next theorem is the main result of the section.

THEOREM 3.3. Let $R$ be a left perfect ring. Then the following conditions are equivalent:

(i) $R$ is a left S-ring;

(ii) $Z(A) \neq 0$ for every proper idempotent ideal $A$ of $R$;

(iii) every projective and faithful left R-module is completely 
faithfuz;

(iv) every finitely generated, projective and faithful left R-module is completely faithful;

(v) $Z(A) \neq 0$ for every proper idempotent ideal $A$ of $R$ of the form $(e\rangle$, for an idempotent element $e \in R$.

Proof. From previous results, it follows that $(i) \Rightarrow(i i) \Rightarrow(i i i) \Rightarrow(i v) \Rightarrow(v)$ for any ring $R$. . (That $(i v) \Rightarrow(v)$ follows from Proposition 3.2.)

Michler [14, Proposition 2.1] has shown that if $R$ is left perfect, then every idempotent ideal of $R$ is of the form $\langle e\rangle$ for some idempotent element $e$ of $R$. Thus $(v) \Rightarrow$ (ii) if $R$ is left perfect. Again if we assume that $R$ is left perfect, then $(i i) \Rightarrow(i)$ by applying Proposition 2.4 and the correspondence between proper cotorsion radicals of $R^{M}$ and proper idempotent ideals of $R$. This completes the proof. //

Certain of the conditions in the above theorem are equivalent in more general circumstances. For instance, (ii) and (iii) are equivalent if $R$ is left hereditary. If $R$ is left hereditary and left noetherian, then conditions (ii) - (iv) are equivalent, a result of Endo [7, Corollary 6.3]. Conditions ( $i$ ) - (ii) are equivalent whenever the intersection of dense right ideals is dense.

We conclude with an observation giving some conditions under which every non-zero projective module is completely faithful. Recall that a left and right hereditary, left and right noetherian prime ring which is a maximal order in its quotient ring is said to be a Dedekind prime ring.

PROPOSITION 3.4. Let $R$ be hereditary and noetherian. The following conditions are equivalent:

(i) $R^{M}$ has no proper cotorsion radicals;

(ii) every non-zero projective $R$-module is completely faithful;

(iii) $R$ is a Dedekind prime ring.

Proof. (i) $\Leftrightarrow$ (ii). Conditions (i) and (ii) are both equivalent to the condition that $R$ has no proper idempotent ideals, since $R$ is left and right hereditary. 
(ii) $\Leftrightarrow$ (iii). Each non-zero ideal $A$ of $R$ is projective, so if condition ( $i$ ) holds, then every ideal $A$ is completely faithful, and thus $Z(A) \neq 0$. This shows that assuming ( $i i$ ) implies that $R$ is a prime ring. The result then follows from Theorem 1.2 of Eisenbud and Robson [6] which states that an hereditary, noetherian prime ring $R$ is a Dedekind prime ring if and only if $R$ has no proper idempotent ideals. //

\section{References}

[0] J.S. Alin and E.P. Armendariz, "TTF-classes over perfect rings", $J$. Austral. Math. Soc. 11 (1970), 499-503.

[1] Maurice Auslander and Oscar Goldman, "Maximal orders", Trans. Amer. Math. Soc. 97 (1960), 1-24.

[2] Goro Azumaya, "Completely faithful modules and self-injective rings", Nagoya Math. J. 27 (1966), 697-708.

[3] Hyman Bass, "Finitistic dimension and a homological generalization of semi-primary rings", Trans. Amer. Math. Soc. 95 (1960), 466-488.

[4] John A. Beachy, "Generating and cogenerating structures", Trans. Amer. Math. Soc. 158 (1971), (to appear).

[5] Vlastimil Dlab, "A characterization of perfect rings", Pacific $J$. Math. 33 (1970), 79-88.

[6] David Eisenbud and J.C. Robson, "Hereditary Noetherian prime rings", J. AZgebra 16 (1970), 86-104.

[7] Shizuo Endo, "Completely faithful modules and quasi-Frobenius algebras", J. Math. Soc. Jopan 19 (1967), 437-456.

[8] Oscar Goldman, "Rings and modules of quotients", J. Algebra 13 (1969), 10-47.

[9] J.P. Jans, "Some aspects of torsion", Pacific J. Math. 15 (1965), 1249-1259.

[10] Yoshiki Kurata, "On an $n$-fold torsion theory in the category $R^{M "}$, J. Algebra (to appear). 
[11] J. Lambek, Lectures on rings and modules (Blaisdell, Waltham, Massachusetts; 1966).

[12] Joachim Lambek, Torsion theories, additive semantics, and rings of quotients (Lecture Notes in Mathematics, No. 177. Springer-Verlag, Berlin, Heidelberg, New York, 1971).

[13] J.-M. Maranda, "Injective structures", Trans. Amer. Math. Soc. 110 (1964), 98-135.

[14] Gerhard Michler, "Idempotent ideals in perfect rings", Conad. $J$. Math. 21 (1969), 301-309.

[15] Kiiti Morita, "On S-rings in the sense of F. Kasch", Nagoya J. Math. 27 (1966), 687-695.

Northern IIlinois University,

DeKalb,

IIIinois, USA. 